\title{
Cochrane
}

Library

Cochrane Database of Systematic Reviews

\section{Melatonin for women in pregnancy for neuroprotection of the fetus} (Review)

Wilkinson D, Shepherd E, Wallace EM

Wilkinson D, Shepherd E, Wallace EM.

Melatonin for women in pregnancy for neuroprotection of the fetus.

Cochrane Database of Systematic Reviews 2016, Issue 3. Art. No.: CD010527.

DOI: 10.1002/14651858.CD010527.pub2.

www.cochranelibrary.com 
ABSTRACT 1

PLAIN LANGUAGE SUMMARY

BACKGROUND

OBJECTIVES

METHODS

RESULTS

Figure 1.

DISCUSSION

AUTHORS' CONCLUSIONS

ACKNOWLEDGEMENTS

REFERENCES

CHARACTERISTICS OF STUDIES

APPENDICES

CONTRIBUTIONS OF AUTHORS

DECLARATIONS OF INTEREST

SOURCES OF SUPPORT

DIFFERENCES BETWEEN PROTOCOL AND REVIEW

INDEX TERMS

\section{TABLE OF CONTENTS}


[Intervention Review]

\section{Melatonin for women in pregnancy for neuroprotection of the fetus}

Dominic Wilkinson ${ }^{1}$, Emily Shepherd ${ }^{2}$, Euan M Wallace ${ }^{3}$

1Oxford Uehiro Centre for Practical Ethics, University of Oxford, Oxford, UK. ${ }^{2}$ ARCH: Australian Research Centre for Health of Women and Babies, Robinson Research Institute, Discipline of Obstetrics and Gynaecology, The University of Adelaide, Adelaide, Australia. ${ }^{3}$ The Ritchie Centre, Monash University, Melbourne, Australia

Contact: Dominic Wilkinson, Oxford Uehiro Centre for Practical Ethics, University of Oxford, Oxford, UK. dominic.wilkinson@philosophy.ox.ac.uk.

Editorial group: Cochrane Pregnancy and Childbirth Group.

Publication status and date: New, published in Issue 3, 2016.

Citation: Wilkinson D, Shepherd E, Wallace EM. Melatonin for women in pregnancy for neuroprotection of the fetus. Cochrane Database of Systematic Reviews 2016, Issue 3. Art. No.: CD010527. DOI: 10.1002/14651858.CD010527.pub2.

Copyright ( 2016 The Cochrane Collaboration. Published by John Wiley \& Sons, Ltd.

\section{A B S T R A C T}

\section{Background}

Melatonin is an antioxidant with anti-inflammatory and anti-apoptotic effects. Animal studies have supported a fetal neuroprotective role for melatonin when administered maternally. It is important to assess whether melatonin, given to the mother, can reduce the risk of neurosensory disabilities (including cerebral palsy) and death, associated with fetal brain injury, for the preterm or term compromised fetus.

\section{Objectives}

To assess the effects of melatonin when used for neuroprotection of the fetus.

\section{Search methods}

We searched the Cochrane Pregnancy and Childbirth Group's Trials Register (31 January 2016).

\section{Selection criteria}

We planned to include randomised controlled trials and quasi-randomised controlled trials comparing melatonin given to women in pregnancy (regardless of the route, timing, dose and duration of administration) for fetal neuroprotection with placebo, no treatment, or with an alternative agent aimed at providing fetal neuroprotection. We also planned to include comparisons of different regimens for administration of melatonin.

\section{Data collection and analysis}

Two review authors planned to independently assess trial eligibility, trial quality and extract the data.

\section{Main results}

We found no randomised trials for inclusion in this review. One study is ongoing.

\section{Authors' conclusions}

As we did not identify any randomised trials for inclusion in this review, we are unable to comment on implications for practice at this stage.

Although evidence from animals studies has supported a fetal neuroprotective role for melatonin when administered to the mother during pregnancy, no trials assessing melatonin for fetal neuroprotection in pregnant women have been completed to date. However, there is currently one ongoing randomised controlled trial (with an estimated enrolment target of 60 pregnant women) which examines the dose of 
melatonin, administered to women at risk of imminent very preterm birth (less than 28 weeks' gestation) required to reduce brain damage in the white matter of the babies that were born very preterm.

Further high-quality research is needed and research efforts should directed towards trials comparing melatonin with either no intervention (no treatment or placebo), or with alternative agents aimed at providing fetal neuroprotection (such as magnesium sulphate for the very preterm infant). Such trials should evaluate maternal and infant short- and longer-term outcomes (including neurosensory disabilities such as cerebral palsy), and consider the costs of care.

\section{PLAIN LANGUAGE SUMMARY}

\section{Giving melatonin to women in pregnancy to protect the baby's brain and improve long-term outcomes for the baby}

\section{What is the issue?}

As a baby's brain develops during pregnancy, it is susceptible to damage. A number of factors can increase the risk of injury to the developing brain of both preterm (before 37 weeks of pregnancy) and term (after 37 weeks of pregnancy) babies. This injury can lead to death of the baby, or if the baby survives, to life-long health problems, such as hearing, sight and speech disorders; seizures; intellectual disabilities; and motor impairments, including cerebral palsy, which is the most common physical disability in childhood. Being born preterm, small-for-gestational age, or low birthweight; abnormalities of the mother's placenta (the organ that connects the developing fetus to the uterine wall); uterine infection; and birth asphyxia, caused by a lack of oxygen to the baby that lasts long enough to cause harm, can increase the risk of brain injury and cerebral palsy with associated impairments or disabilities.

Melatonin is a small hormone that is usually made by the pineal gland (a small gland in the brain) during the night. It helps to regulate the sleep-wake cycle, is an antioxidant (that protect cells from the damage caused by free radicals), and interacts with the immune system (the structures and processes in the body that protect against disease).

\section{Why is this important?}

It is possible that melatonin, given to a mother in pregnancy, can help protect her baby's brain. Animal studies, including in mice, rats and sheep, have suggested that melatonin may be able to protect the developing human baby's brain from injury when given to the mother during pregnancy.

\section{What evidence did we find?}

We did not find any completed randomised controlled trials that assessed melatonin given to the mother during pregnancy to help protect the baby's brain. One ongoing trial (planning to include 60 women) was identified. This trial is designed to determine what dose of melatonin can reduce brain injury for babies when given to their mothers before very preterm birth (before 28 weeks of pregnancy).

\section{What does this mean?}

Further studies are needed to establish whether melatonin given to the mother in pregnancy can protect the baby's brain against brain injury. The babies in these trials need to be followed up over a long period so that we can monitor the effects of melatonin on childhood development, including impairments or disabilities such as cerebral palsy. 


\section{B A C K G R O U N D}

\section{Description of the condition}

\section{Fetal brain injury: risk factors and consequences}

Injury to the fetal brain is a major contributor to morbidity and mortality in infants and children born preterm (at less than 37 weeks' gestation) and at term (at 37 weeks' gestation and later) (Vexler 2001; Volpe 2000). The pathogenesis of brain injury is known to be complex and multifactorial, with a number of interrelated pathways contributing to central nervous system cellular dysfunction and death, including the accumulation of reactive oxygen species, the release of excitatory amino acids, energy depletion and apoptosis (Inder 2000; Vexler 2001). While there are multiple causes of such brain injury, including hypoxiaischaemia (characterised by a reduction of oxygen in the blood combined with reduced blood flow to the brain), haemorrhage, infection and metabolic derangement, hypoxia-ischaemia is believed to be an important cause of brain injury in a large number of cases (Volpe 2000). Brain hypoxia (deficiency of oxygen) and ischaemia (insufficient blood supply) may lead to different neuropathology in infants born prematurely and at term, with neuronal cell injury predominating in term infants, and cerebral white matter injury predominating in premature infants (Volpe 2000). Injury to the developing brain is known to be associated with a number of long-term sequelae for the infant and child, including hearing, sight and speech disorders, seizures, intellectual disabilities, and motor impairments, such as cerebral palsy (Vexler 2001).

Cerebral palsy is a broad term, encompassing non-progressive (but not unchanging) permanent physical disorders of movement or posture acquired in early life, resulting from complications in brain development (interferences, lesions or abnormalities) (ACPR Group 2009; Blair 2006). Cerebral palsy is the most frequent cause of childhood motor disability, affecting approximately two per 1000 live births in high-income countries (ACPR Group 2009). It is estimated that in approximately $94 \%$ of cases (13 in 14), the brain injury leading to cerebral palsy occurs to the fetus in utero, or before one month of age (ACPR Group 2009). Other neurologic impairments and neurosensory disabilities frequently associated with cerebral palsy include hearing, sight and speech disorders, intellectual disability and epilepsy.

Preterm birth is one of the principal risk factors for cerebral palsy and associated neurologic impairments and neurosensory disabilities (Blair 2006; Drummond 2002; Himpens 2008). The degree of prematurity is associated with vulnerability of cerebral white matter, and is predictive of an increasing risk of white matter injury such as periventricular leukomalacia, and of intraventricular haemorrhage (Larroque 2003) - established risk factors for the development of cerebral palsy and associated neurosensory disabilities (Saliba 2001). Although preterm birth is acknowledged as a major risk factor for cerebral palsy (ACPR Group 2009), accounting for approximately $40 \%$ of all cases, most children with cerebral palsy are born at term (ACPR Group 2009; Wu 2003).

Along with very preterm birth, low birthweight is an independent, important risk factor for cerebral palsy and associated neurosensory disabilities, particularly in infants born at term or moderately preterm (Blair 1990). A number of studies have revealed high risks of neurosensory impairments and disabilities (including cerebral palsy, blindness, deafness and intellectual impairment) for very low birthweight (less than $1500 \mathrm{~g}$ ) and extremely low birthweight (less than $1000 \mathrm{~g}$ ) infants, when compared to normal birthweight controls (Doyle 2001). Intrauterine growth restriction and being small-for-gestational age at birth have additionally been shown to be important risk factors for neurologic injury and longterm sequale for both preterm and term fetuses (Jacobsson 2008; Leitner 2000; Low 1992; O'Keeffe 2003). For term infants, perinatal asphyxia (a condition resulting from deprivation of oxygen to a newborn infant, lasting long enough to cause physical harm) has been shown to be an important cause of brain injury and later neurodevelopmental disabilities (Dilenge 2001; Greenwood 2005).

Though conflicting evidence exists, other proposed risk factors for neurologic injury and associated longer-term consequences (including cerebral palsy) include increasing plurality (with pregnancies of higher plurality being more likely to be of shorter gestation and associated with slower uterine growth) (Blair 2006), infection such as chorioamnionitis (Blair 2006; Greenwood 2005; Wu 2003), and pre-eclampsia (Blair 2006; Greenwood 2005).

While a great number of potential risk factors for neurosensory disabilities have been identified, their commonality is that separately, or in combination, they may lead to fetal brain injury. The aim of primary preventative strategies, therefore, is to target early stages in the multifactorial, interrelated pathways before brain injury becomes irreversible.

\section{Description of the intervention \\ Melatonin}

Melatonin (N-acetyl-5-methoxytryptamin) is a small lipid-soluble hormone that is primarily synthesised and secreted by the pineal gland at night, under normal environmental conditions (Claustrat 2005); it has been recognised as a "ubiquitously distributed and functionally diverse molecule" (Reiter 2010). The key physiological functions of melatonin include mediating seasonality and circadian rhythm (Claustrat 2005). The endogenous rhythm of melatonin secretion is related to the light-dark cycle, however, the regulating system of secretion is complex, following both central and autonomic pathways (Claustrat 2005). There are thus many pathophysiological situations where melatonin secretion can be disturbed, and alterations of regulation can increase predisposition to disease, and modify the courses and outcomes of disorders (Claustrat 2005; Reiter 2010).

A feature that characterises melatonin is the variety of mechanisms that it employs to modulate the physiology and molecular biology of cells (Reiter 2010). In addition to mediating action through wellcharacterised G-protein coupled melatonin receptors in cellular membranes, melatonin is able to detoxify oxygen free radicals and related oxygen derivatives, and thus can also influence cellular physiology via receptor-indepedent means (Reiter 2010). The complexities of such processes have made it at times difficult to determine how melatonin functions to exert particular actions (Reiter 2010). Despite this, melatonin has been regarded as a molecule with "virtual absence of toxicity" (Reiter 2010), with great potential for application in human medicine, due to its ability to contribute to improved cellular physiology.

Clinically, melatonin has been shown to be effective in preventing and reducing jet lag (Herxheimer 2002), and some benefits have been seen when it is given for dementia, with a reduction 
in dementia-related psychopathologic behaviour disturbances observed (Jansen 2006). Melatonin has also been used to treat non-respiratory related sleep disorders in children, though benefits for this indication are currently not confirmed (Khan 2011; Khan 2011b). Melatonin has been shown to reduce oxidative stress in human newborns with septicaemia, and lead to improved clinical outcomes (Gitto 2001). It has also been shown to reduce products associated with oxidative stress and damage in the blood of asphyxiated human newborns (Fulia 2001).

\section{How the intervention might work}

\section{Melatonin for fetal neuroprotection}

While the pathogenesis of white matter brain injury (such as periventricular leukomalacia), associated with perinatal mortality and long-term neurosensory disabilities, is complex and multifactorial, free radical-induced oxidative damage and infection or inflammatory-induced damage appear to be important. Free radical-induced damage also appears to be important in neonatal haemorrhagic brain injury (such as intraventricular haemorrhage) (Lekic 2011), which may subsequently lead to neurosensory disabilities, including cerebral palsy.

In adult animals, melatonin has been shown to be neuroprotective in models of focal cerebral ischaemia (stroke) (Macleod 2005), and in neonatal mice, melatonin has been shown to attenuate the development of white matter cysts following acute excitotoxic brain injury (Husson 2002). More recently, the neuroprotective effects of melatonin in the fetal brain have been assessed. Following intrauterine asphyxia (via umbilical cord occlusion), melatonin administration to both preterm and near-term fetal sheep has been shown to reduce oxidative stress (Miller 2005) and attenuate cell death (including apoptosis) in the fetal brain, in association with a reduced inflammatory response (Welin 2007). Systemic administration of melatonin following acute neonatal haemorrhagic brain injury in rats, has been shown to protect against post-haemorrhagic consequences of brain atrophy, splenomegaly and cardiac hypertrophy (Lekic 2011). Importantly, melatonin has been shown to improve functional outcomes following such brain injury - ameliorating cognitive and sensorimotor dysfunction in the juvenile rat (Lekic 2011).

In addition to the direct fetal and neonatal administration of melatonin being associated with neuroprotective effects, the maternal administration of melatonin has been shown benefit the offspring. The prophylactic administration of melatonin to pregnant rats immediately prior to an acute ischaemic episode (Watanabe 2004) and regularly throughout pregnancy (Watanabe 2012), has been shown to reduce ischaemia-reperfusion-induced oxidative damage in the premature fetal rat brain (Watanabe 2004; Watanabe 2012). Maternal administration of melatonin one hour after an ischaemic episode has also been shown to prevent against ischaemia-reperfusion-induced oxidative brain damage and degeneration in neonatal rats (Hamada 2010). At three to 12 hours post reperfusion however, melatonin has failed to protect against oxidative brain injury in neonatal rats (Hamada 2010), suggesting that there may be a critical window of time to administer melatonin, after which the molecule no longer has therapeutic benefit. Further, maternal administration of melatonin in an ovine model of fetal growth restriction has been shown to reduce brain oxidative stress, restore normal white matter myelination, and improve motor and cognitive function in the growth restricted lambs (Miller 2014). Others have similarly shown that melatonin protects against white matter injury in a rodent model of fetal growth restriction (when administered to rat pups after birth) (Olivier 2009).

In view of the evidence from such studies, it is plausible that melatonin, an antioxidant with apparent anti-inflammatory and anti-apoptotic effects (Cheung 2003), may protect the human fetal brain against free radical-induced brain damage occurring during times of increased oxidative stress in pregnancy (such as in pregnancies complicated by pre-eclampsia, intrauterine growth restriction, infection, and preterm delivery) when administered fetally or maternally.

\section{Why it is important to do this review}

Melatonin is an antioxidant with anti-inflammatory and antiapoptotic effects. Animal studies have supported a fetal neuroprotective role for melatonin when administered maternally. It is important to assess whether melatonin, when given to the mother, may reduce the risk of neurosensory disabilities (including cerebral palsy) and death, for the preterm or term compromised fetus.

\section{O B J E C T IVES}

To assess the effects of melatonin when used for neuroprotection of the fetus.

\section{METHODS}

\section{Criteria for considering studies for this review}

\section{Types of studies}

We planned to include all published, unpublished and ongoing randomised trials and quasi-randomised trials assessing melatonin for fetal neuroprotection. We planned to exclude cross-over and cluster-randomised trials. We planned to include studies reported as abstracts only.

\section{Types of participants}

We planned to include any pregnant woman administered melatonin, regardless of whether the pregnancy was single or multiple, and regardless of the gestation period at which melatonin was given. This could have included trials where melatonin was given as the fetus was preterm or growth restricted, for chorioamnionitis, for prelabour rupture of membranes, for antenatal/intrapartum fetal distress, for pre-eclampsia, or other reasons.

\section{Types of interventions}

We planned to include all comparisons of melatonin given to women, with a placebo or no treatment, or to an alternative agent aimed at providing fetal neuroprotection. We planned to also include comparisons of different regimens for administration of melatonin. We planned to include studies regardless of the route (i.e. oral, intramuscular or intravenous), timing, dose and duration of melatonin administration. 


\section{Types of outcome measures}

\section{Primary outcomes}

Primary outcomes were chosen to be most representative of the clinically important measures of effectiveness and safety, including serious outcomes and adverse effects.

\section{For the infant/child}

- Death or any neurosensory disability (at latest time reported) (this combined outcome recognises the potential for competing risks of death or survival with neurological problems)

- Death (defined as all fetal, neonatal or later death) (at latest time reported)

- Neurosensory disability (any of cerebral palsy, blindness, deafness, developmental delay/intellectual impairment) (at latest time reported)

\section{Definitions}

- Cerebral palsy: abnormality of tone with motor dysfunction (as diagnosed at 18 months of age or later)

- Blindness: corrected visual acuity worse than $6 / 60$ in the better eye

- Deafness: hearing loss requiring amplification or worse

- Developmental delay/intellectual impairment: a standardised score less than minus one standard deviation (SD) below the mean

\section{For the mother}

- Any adverse effects severe enough to stop treatment

\section{Secondary outcomes}

Secondary outcomes include other measures of effectiveness and safety.

\section{For the fetus/infant}

- Abnormal fetal and umbilical Doppler ultrasound study (as defined by trialists)

- Fetal death

- Neonatal death

- Gestational age at birth

- Birthweight (absolute and centile)

- Apgar score (less than seven at five minutes)

- Active resuscitation via an endotracheal tube at birth

- Use and duration of respiratory support (mechanical ventilation or continuous positive airways pressure, or both)

- Intraventricular haemorrhage (including severity - grade one to four) (as defined by trialists)

- Periventricular leukomalacia (as defined by trialists)

- Hypoxic ischaemic encephalopathy (as defined by trialists)

- Neonatal encephalopathy (as defined by trialists)

- Proven neonatal sepsis

- Necrotising enterocolitis

- Abnormal neurological examination (however defined by the trialists, at a point earlier than 18 months of age)

\section{For the mother}

- Side effects and serious adverse events associated with treatment

- Maternal white cell count and platelet count

- Women's satisfaction with the treatment

- Mode of birth (normal vaginal birth, operative vaginal birth, caesarean section), and indication for non-elective mode of birth

\section{For the infant/child}

- Cerebral palsy (any, and graded as severe: including children who are non-ambulant and are likely to remain so; moderate: including those children who have substantial limitation of movement; mild: including those children walking with little limitation of movement)

- Death or cerebral palsy

- Blindness

- Deafness

- Developmental delay/intellectual impairment (classified as severe: a developmental quotient or intelligence quotient less than minus three SD below the mean; moderate: a developmental quotient or intelligence quotient from minus three SD to minus two SD below the mean; mild: a developmental quotient or intelligence quotient from minus two SD to minus one SD below the mean)

- Major neurosensory disability (defined as any of: moderate or severe cerebral palsy, legal blindness, neurosensory deafness requiring hearing aids, or moderate or severe developmental delay/intellectual impairment)

- Death or major neurosensory disability

- Growth assessments at childhood follow-up (weight, head circumference, length/height)

\section{Use of health services}

- Admission to intensive care unit for the mother

- Length of postnatal hospitalisation for the women

- Admission to neonatal intensive care for the infant and length of stay

- Costs of care for the mother or infant, or both

\section{Search methods for identification of studies}

The following methods section of this review is based on a standard template used by the Cochrane Pregnancy and Childbirth Group.

\section{Electronic searches}

We searched the Cochrane Pregnancy and Childbirth Group's Trials Register by contacting the Trials Search Co-ordinator (31 January 2016).

The Register is a database containing over 20,000 reports of controlled trials in the field of pregnancy and childbirth. For full search methods used to populate the Pregnancy and Childbirth Group's Trials Register including the detailed search strategies for CENTRAL, MEDLINE, Embase and CINAHL; the list of handsearched journals and conference proceedings, and the list of journals reviewed via the current awareness service, please follow this link to the editorial information about the Cochrane Pregnancy and Childbirth Group in The Cochrane Library and select the 
'Specialized Register' section from the options on the left side of the screen.

Briefly, the Cochrane Pregnancy and Childbirth Group's Trials Register is maintained by the Trials Search Co-ordinator and contains trials identified from:

1. monthly searches of the Cochrane Central Register of Controlled Trials (CENTRAL);

2. weekly searches of MEDLINE (Ovid);

3. weekly searches of Embase (Ovid);

4. monthly searches of CINAHL (EBSCO);

5. handsearches of 30 journals and the proceedings of major conferences;

6. weekly current awareness alerts for a further 44 journals plus monthly BioMed Central email alerts.

Search results are screened by two people and the full text of all relevant trial reports identified through the searching activities described above is reviewed. Based on the intervention described, each trial report is assigned a number that corresponds to a specific Pregnancy and Childbirth Group review topic (or topics), and is then added to the Register. The Trials Search Co-ordinator searches the Register for each review using this topic number rather than keywords. This results in a more specific search set which has been fully accounted for in the relevant review sections (Included, Excluded, Awaiting Classification or Ongoing).

\section{Searching other resources}

We planned to search reference lists of retrieved studies.

We did not apply any language or date restrictions.

\section{Data collection and analysis}

See Appendix 1 for methods of data collection and analysis to be used in future updates of this review.

\section{RES U LTS}

\section{Description of studies}

\section{Results of the search}

The search of the Cochrane Pregnancy and Childbirth Group's Trials Register retrieved one trial report (Figure 1). This trial was identified as ongoing, and aims to recruit 60 women at risk of imminent very preterm birth ( 28 weeks' gestation), to determine the dose of melatonin, administered to women prior to birth, required to reduce white matter brain damage in the infants born very preterm (Valerie 2015). See Characteristics of ongoing studies for further details. 
Figure 1. Study flow diagram.

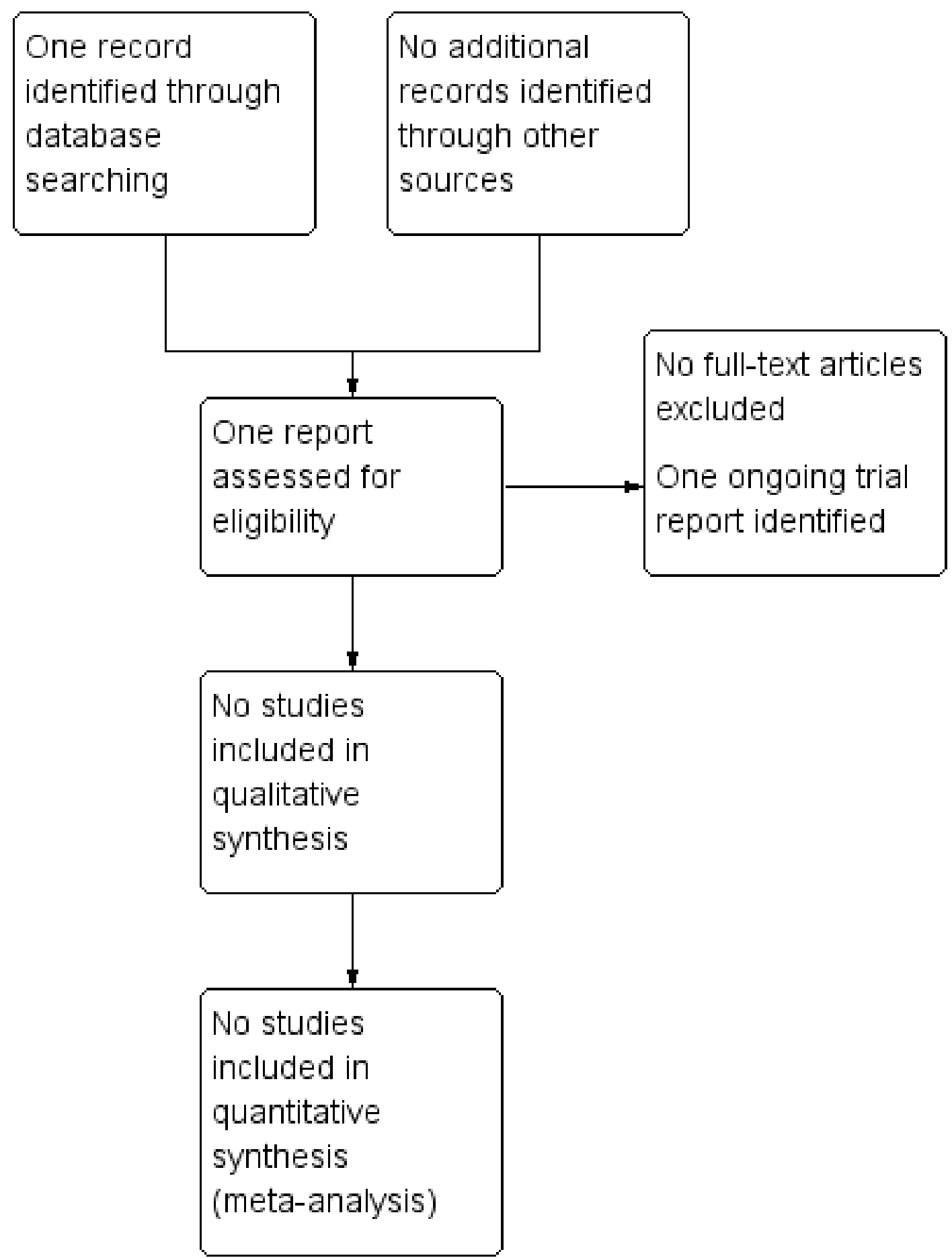

\section{Risk of bias in included studies}

No randomised trials were found for inclusion in this review.

\section{Effects of interventions}

No randomised trials were found for inclusion in this review.

\section{DISCUSSION}

There were no completed randomised controlled trials identified assessing the benefits and harms of melatonin for women in pregnancy for neuroprotection of the fetus.

Death and neurosensory disabilities, such as cerebral palsy, are serious outcomes after a preterm or term compromised birth, and thus the identification of primary preventative therapies, is of crucial importance. 
Compelling evidence from recent and extensive animal studies has provided promise that melatonin could potentially be a simple and effective neuroprotective strategy for the fetus, infant and child (Balduini 2012; Husson 2002; Lekic 2011; Macleod 2005; Miller 2005; Olivier 2009; Robertson 2013; Welin 2007), including when administered maternally (Drury 2014; Hamada 2010; Hutton 2009; Miller 2014; Watanabe 2004; Watanabe 2012; Yawno 2012). Melatonin has been shown to be a powerful antioxidant (Aversa 2012), easily crosses most biological cell membranes including the placenta (Miller 2005; Okatani 1998) and blood-brain barrier (Welin 2007), and has not been shown to pose serious risks for the mother or her fetus, including when administered at high doses (Jahnke 1999).

In the absence of randomised controlled trial data on which to base clinical decisions, uncertainty however, persists about the relative benefits and harms of melatonin when given to women in pregnancy for fetal neuroprotection. A recent commentary of antenatal and postnatal neuroprotective agents "ready for bench to bedside translation for the newborn infant" highlighted that of 13 potential agents identified, melatonin was the second choice of potential antenatal therapies to be given to a mother in whom fetal distress is detected, and was the first choice of potential postnatal therapies to be given as a rescue therapy in moderate to severe neonatal encephalopathy (Robertson 2012). Antenatal treatments were ranked based on consideration of: placenta transfer; ease of administration; knowledge about starting dose; adverse effects; teratological or toxic effects; overall benefit and efficacy. The authors of that commentary highlighted the "remarkable safety, efficacy, and ease of administration of this medication, [suggesting] that melatonin has strong potential to be translated to the clinic for clinical trials" (Robertson 2012).

One such pilot study is underway in Australia, assessing whether melatonin supplementation throughout pregnancies complicated by intrauterine growth restriction can reduce oxidative stress experienced by the fetus, and thus prevent fetal brain injury (Alers 2013). Another pilot study in Australia is currently assessing whether maternal administration of melatonin in preterm pregnancies complicated by pre-eclampsia, can decrease the level of oxidative stress (Hobson 2013).

This review identified one ongoing randomised controlled trial, which aims to recruit 60 women at risk of imminent, very preterm birth, to determine the dose of melatonin required to reduce white matter brain injury in the infants born very preterm (Valerie 2015). Larger trials, powered to detect differences in short- and longer-term maternal and infant health outcomes, will however be required in order to further address questions surrounding effectiveness and safety.

\section{AUTHORS'CONCLUSIONS}

\section{Implications for practice}

As we did not identify any eligible trials for inclusion in this review, we are unable to comment on implications for practice regarding the use of melatonin for women in pregnancy for neuroprotection of the fetus.

\section{Implications for research}

The available animal studies of melatonin in pregnancy for fetal neuroprotection provide some insight into the potential benefits of this intervention. Randomised controlled trials are required, however, to provide the most reliable evidence regarding any potential benefits and harms of giving pregnant women melatonin for neuroprotection of the fetus. One small randomised controlled trial is currently underway.

Research efforts should first be directed towards trials comparing melatonin with either no intervention (no treatment or placebo), or with an alternative agent aimed at providing fetal neuroprotection (such as magnesium sulphate for the very preterm infant); followed by trials comparing different melatonin regimens, should melatonin be shown to be effective and safe. Such trials must be of a high quality, and of a sufficient sample size to assess the comparative effects on fetal, infant and child mortality and morbidity such as cerebral palsy and other neurosensory disabilities, maternal outcomes including adverse effects, and the use of health services.

\section{ACK N OWLEDGEMENTS}

As part of the pre-publication editorial process, this review has been commented on by two peers (an editor and referee who is external to the editorial team), a member of the Pregnancy and Childbirth Group's international panel of consumers and the Group's Statistical Adviser.

This project was supported by the National Institute for Health Research, via Cochrane Infrastructure funding to Cochrane Pregnancy and Childbirth. The views and opinions expressed therein are those of the authors and do not necessarily reflect those of the Systematic Reviews Programme, NIHR, NHS or the Department of Health.

We thank Caroline Crowther and Philippa Middleton for their advice on the development of the protocol for this review. 


\section{REFERE N CES}

\section{References to ongoing studies}

Valerie 2015 \{published data only\}

Valerie B. Therapeutic effects of maternal melatonin administration on brain injury and white matter disease (PREMELIP). ClinicalTrials.gov (http://clinicaltrials.gov/) [accessed 14 May 2015] 2015.

\section{Additional references}

\section{ACPR Group 2009}

Australian Cerebral Palsy Register (ACPR) Group. Report of the Australian Cerebral Palsy Register, Birth Years 1993-2003. http:// www.cpregister.com/ (accessed 16 October 2012).

\section{Alers 2013}

Alers NO, Jenkin G, Miller SL, Wallace EM. Antenatal melatonin as an antioxidant in human pregnancies complicated by fetal growth restriction - a phase I pilot clinical trial: study protocol. BMJ Open 2013;3(12):e004141.

\section{Aversa 2012}

Aversa S, Pellegrino S, Barberi I, Reiter RJ, Gitto E. Potential utility of melatonin as an antioxidant during pregnancy and in the perinatal period. Journal of Maternal-Fetal and Neonatal Medicine 2012;25(3):207-21.

\section{Balduini 2012}

Balduini W, Carloni S, Perrone S, Bertrando S, Tataranno ML, Negro S, et al. The use of melatonin in hypoxic-ischemic brain damage: an experimental study. Journal of Maternal-Fetal and Neonatal Medicine 2012;25(Suppl 1):119-24.

\section{Blair 1990}

Blair E, Stanley F. Intrauterine growth and spastic cerebral palsy. I. Association with birth weight for gestational age. American Journal of Obstetrics and Gynecology 1990;162(1):229-37.

\section{Blair 2006}

Blair E, Watson L. Epidemiology of cerebral palsy. Seminars in Fetal and Neonatal Medicine 2006;11:117-25.

\section{Cheung 2003}

Cheung RTF. The utility of melatonin in reducing cerebral damage resulting from ischemia and reperfusion. Journal of Pineal Research 2003;34(3):153-60.

\section{Claustrat 2005}

Claustrat B, Brun J, Chazot G. The basic physiology and pathophysiology of melatonin. Sleep Medicine Reviews 2005;9(1):11-24.

\section{Dilenge 2001}

Dilenge M, Majnemer A, Shevell M. Long-term developmental outcome of asphyxiated term neonates. Journal of Child Neurology 2001;16:781-92.

\section{Doyle 2001}

Dolye LW, Casalaz D, Victorian Infant Collaborative Study Group. Outcome at 14 years of extremely low birthweight infants: a regional study. Archives of Disease in Childhood. Fetal and Neonatal Edition 2001;85:F159-F164.

\section{Drummond 2002}

Drummond PM, Colver AF. Analysis by gestational age of cerebral palsy in singleton births in north-east England 1970-94. Paediatric and Perinatal Epidemiology 2002;16(2):172-80.

\section{Drury 2014}

Drury PP, Davidson JO, Bennet L, Booth LC, Tan S, Fraser M, et al. Partial neural protection with prophylactic low-dose melatonin after asphyxia in preterm fetal sheep. Journal of Cerebral Blood Flow and Metabolism 2014;34(1):126-35.

\section{Fulia 2001}

Fulia F, Gitto E, Cuzzocrea S, Reiter RJ, Dugo L, Gitto P, et al. Increased levels of malondialdehyde and nitrite/nitrate in the blood of asphyxiated newborns: reduction by melatonin. Journal of Pineal Research 2001;31(4):343-9.

\section{Gates 2004}

Gates S, Brocklehurst P. How should trials recruiting women with multiple pregnancies be analysed?. British Journal of Obstetrics and Gynaecology 2004;111:213-9.

\section{Gitto 2001}

Gitto E, Karbownik M, Reiter RJ, Tan DX, Cuzzocrea S, Chiurazzi P, et al. Effects of melatonin treatment in septic newborns. Pediatric Research 2001;50(6):756-60.

\section{Greenwood 2005}

Greenwood C, Yudkin P, Sellers S, Impey L, Doyle P. Why is there a modifying effect of gestational age on risk factors for cerebral palsy?. Archives of Disease in Childhood. Fetal and Neonatal Edition 2005;90(2):F141-F146.

\section{Hamada 2010}

Hamada F, Watanabe K, Wakatsuki A, Nagai R, Shinohara K, Hayashi $Y$, et al. Therapeutic effects of maternal melatonin administration on ischemia/reperfusion-induced oxidative cerebral damage in neonatal rats. Neonatology 2010;98(1):33-40.

\section{Herxheimer 2002}

Herxheimer A, Petrie KJ. Melatonin for the prevention and treatment of jet lag. Cochrane Database of Systematic Reviews 2002, Issue 2. [DOI: 10.1002/14651858.CD001520]

\section{Higgins 2011}

Higgins JPT, Green S, editors. Cochrane Handbook for Systematic Reviews of Interventions Version 5.1.0 [updated March 2011]. The Cochrane Collaboration, 2011. Available from www.cochrane-handbook.org. 


\section{Himpens 2008}

Himpens E, Van den Broeck C, Oostra A, Calders P, Vanhaesebrouck P. Prevalence, type, distribution, and severity of cerebral palsy in relation to gestational age: a metaanalytic review. Developmental Medicine and Child Neurology 2008;50(5):334-40.

\section{Hobson 2013}

Hobson SR, Lim R, Gardiner EE, Alers NO, Wallace EM. Phase I pilot clinical trial of antenatal maternally administered melatonin to decrease the level of oxidative stress in human pregnancies affected by pre-eclampsia (PAMPR): study protocol. BMJ Open 2013;3:e003788.

\section{Husson 2002}

Husson I, Mesples B, Bac P, Vamecq J, Evrard P, Gressens P. Melatoninergic neuroprotection of the murine periventricular white matter against neonatal excitotoxic challenge. Annals of Neurology 2002;51:82-92.

\section{Hutton 2009}

Hutton LC, Abbass M, Dickinson H, Ireland Z, Walker DW. Neuroprotective properties of melatonin in a model of birth asphyxia in the spinymouse (Acomys cahirinus). Developmental Neuroscience 2009;31:437-51.

\section{Inder 2000}

Inder TE, Volpe JJ. Mechanisms of perinatal brain injury. Seminars in Neonatology 2000;5:3-16.

\section{Jacobsson 2008}

Jacobsson B, Ahlin K, Francis A, Hagberg G, Hagberg H, Gardosi J. Cerebral palsy and restricted growth status at birth: population-based case-control study. British Journal of Obstetrics and Gynaecology 2008;115(10):1250-5.

\section{Jahnke 1999}

Jahnke G, Marr M, Myers C, Wilson R, Travlos G, Price C. Maternal and developmental toxicity evaluation of melatonin administered orally to pregnant Sprague-Dawley rats. Toxicological Sciences 1999;50:271-9.

\section{Jansen 2006}

Jansen SL, Forbes D, Duncan V, Morgan DG, Malouf R. Melatonin for the treatment of dementia. Cochrane Database of Systematic Reviews 2006, Issue 1. [DOI: 10.1002/14651858.CD003802.pub3]

\section{Khan 2011}

Khan S, Heussler H, McGuire T, Dakin C, Pache D, Cooper D, et al. Melatonin for non-respiratory sleep disorders in visually impaired children. Cochrane Database of Systematic Reviews 2011, Issue 11. [DOI: 10.1002/14651858.CD008473.pub2]

\section{Khan 2011b}

Khan S, Heussler H, McGuire T, Dakin C, Pache D, Cooper D, et al. Melatonin for non-respiratory sleep disorders in typically developing children. Cochrane Database of Systematic Reviews 2011, Issue 5. [DOI: 10.1002/14651858.CD009141]

\section{Larroque 2003}

Larroque B, Marret S, Ancel PY, Arnaud C, Marpeau L, Supernant $\mathrm{K}$, et al. White matter damage and intraventricular hemorrhage in very preterm infants: the EPIPAGE study. Journal of Pediatrics 2003;143(4):477-83.

\section{Leitner 2000}

Leitner Y, Fattal-Valevski A, Geva R, Bassan H, Posner E, Kutai M, et al. Six-year follow-up of children with intrauterine growth retardation: long-term, prospective study. Journal of Child Neurology 2000;15:781-6.

\section{Lekic 2011}

Lekic T, Manaenko A, Rolland W, Virbel K, Hartman R, Tang J, et al. Neuroprotection by melatonin after germinal matrix hemorrhage in neonatal rats. Acta Neurochirurgica 2011;111:201-6.

\section{Low 1992}

Low JA, Handley-Derry MH, Burke SO, Peters RD, Pater EA, Killen $\mathrm{HL}$, et al. Association of intrauterine fetal growth retardation and learning deficits at age 9 to 11 years. American Journal of Obstetrics and Gynecology 1992;167:1499-505.

\section{Macleod 2005}

Macleod MR, O'Collins T, Horky LL, Howells DW, Donnan GA. Systematicreview and meta-analysis of the efficacy of melatonin in experimental stroke. Journal of Pineal Research 2005;38:35-41.

\section{Miller 2005}

Miller SL, Yan EB, Castillo-Melendez M, Jenkin G, Walker DW. Melatonin provides neuroprotection in the late gestation fetal sheep brain in response to umbilical cord occlusion. Developmental Neuroscience 2005;27:200-10.

\section{Miller 2014}

Miller SL, Yawno T, Alers NO, Castillo-Melendez M, Supramaniam VG, VanZyl N, et al. Antenatal antioxidant treatment with melatonin to decrease newborn neurodevelopmental deficits and brain injury caused by fetal growth restriction. Journal of Pineal Research 2014;56(3):283-94.

\section{Okatani 1998}

Okatani Y, Okamoto K, Hayashi K, Wakatsuki A, Tamura S, Sagara Y. Maternal-fetal transfer of melatonin in pregnant women near term. Journal of Pineal Research 1998;25:129-34.

\section{Olivier 2009}

Olivier P, Fontaine RH, Loron G, Van Steenwinckel J, Biran V, Massonneau V. Melatonin promotes oligodendroglial maturation of injured white matter in neonatal rats. PloS One 2009;4(9):e7128.

\section{O'Keeffe 2003}

O'Keeffe MJ, O'Callaghan M, Williams GM, Najman JM, Bor W. Learning, cognitive, and attentional problems in adolescents born small for gestational age. Pediatrics 2002;112:301-7. 


\section{Reiter 2010}

Reiter RJ, Tan DX, Fuentes-Broto L. Melatonin - a multi-tasking molecule. Progress in Brain Research 2010;181:127-51.

\section{RevMan 2014 [Computer program]}

The Nordic Cochrane Centre, The Cochrane Collaboration. Review Manager (RevMan). Version 5.3. Copenhagen: The Nordic Cochrane Centre, The Cochrane Collaboration, 2014.

\section{Robertson 2012}

Robertson NJ, Tan S, Groenendaal F, van Bel F, Juul SE, Bennet $L$, et al. Which neuroprotective agents are ready for bench to bedside translation in the newborn infant?. Journal of Pediatrics 2012;160:544-52.

\section{Robertson 2013}

Robertson NJ, Faulkner S, Fleiss B, Bainbridge A, Andorka C, Price D, et al. Melatonin augments hypothermic neuroprotection in a perinatal asphyxia model. Brain 2013;136(Pt 1):90-105.

\section{Saliba 2001}

Saliba E, Marret S. Cerebral white matter damage in the preterm infant: pathophysiology and risk factors. Seminars in Neonatology 2001;6(2):121-33.

\section{Vexler 2001}

Vexler ZS, Ferriero DM. Molecular and biochemical mechanisms of perinatal brain injury. Seminars in Neonatology 2011;6:99-108.

\section{CHARACTERISTICS OF STUDIES}

Characteristics of ongoing studies [ordered by study ID]

\section{Volpe 2000}

Volpe JJ. Perinatal brain injury: from pathogenesis to neuroprotection. Mental Retardation and Developmental Disabilities Research Reviews 2000;7:56-64.

\section{Watanabe 2004}

Watanabe K, Wakatsuki A, Shinohara K, Ikenoue N, Yokota K, Fukaya T. Maternally administered melatonin protects against ischemia and reperfusion-induced oxidative mitochondrial damage in premature fetal rat brain. Journal of Pineal Research 2004;37(4):276-80.

\section{Watanabe 2012}

Watanabe K, Hamada F, Wakatsuki A, Nagai R, Shinohara K, Hayashi Y, et al. Prophylactic administration of melatonin to the mother throughout pregnancy can protect against oxidative cerebral damage in neonatal rats. Journal of Maternal-Fetal and Neonatal Medicine 2012;25(8):1254-9.

\section{Welin 2007}

Welin AK, Svedin P, Lapatto R, Sultan B, Hagberg H, Gressens P, et al. Melatonin reduces inflammation and cell death in white matter in the mid-gestation fetal sheep following umbilical cord occlusion. Pediatric Research 2007;61(2):153-8.

\section{Wu 2003}

Wu Y, Escobar G, Grether J, Croen L, Greene J, Newman T. Chorioamnionitis and cerebral palsy in term and near-term infants. JAMA 2003;290(20):2677-84.

\section{Yawno 2012}

Yawno T, Castillo-Melendez M, Jenkin G, Wallace EM, Walker DW, Miller SL. Mechanisms of melatonin-induced protection in the brain of late gestation fetal sheep in response to hypoxia. Developmental Neuroscience 2012;34:543-51.

Valerie 2015

Trial name or title
Therapeutic effects of maternal melatonin administration on brain injury and white matter disease (PREMELIP).

Methods Randomised controlled trial.

Participants

Inclusion criteria: women between 24 and 28 weeks' gestation, at imminent risk of preterm birth (cervical dilation greater than or equal to $3 \mathrm{~cm}$ and regular, painful contractions; or booked for elective caesarean section); who are at least 18 years old; have provided written consent; and are joining a security scheme.

Exclusion criteria: outborn deliveries; magnesium sulphate infusion; chronic renal and hepatic impairment before pregnancy; circumstances of maternal or fetal distress requiring emergency caesarean (eclampsia, placental praevia/abruption).

Control: placebo administration to the woman. 
Valerie 2015 (Continued)

Outcomes

Primary: white matter injury at 40 weeks corrected age by brain MRI with diffusion tensor sequence (TBSS analysis).

Secondary: plasma melatonin levels in the mother, and newborn at birth; neurological evaluation at the age of 2 years by the revised Brunet-Lezine test; mortality at 28 days of life and at discharge.

\begin{tabular}{ll}
\hline Starting date & March 2015. \\
\hline Contact information & Biran Valérie: valerie.biran@rdb.aphp.fr \\
& Hôpital Robert-Debré, Paris, France, 75019
\end{tabular}

Notes $\quad$ Estimated study completion: February 2018.

Estimated enrolment: 60 women.

MRI: Magnestic Resonance Imaging

TBSS: Tract-Based Spatial Statistics

\section{AP PE N DICES}

\section{Appendix 1. Methods of data collection and analysis to be used in future updates of this review}

\section{Selection of studies}

Two review authors will independently assess for inclusion all the potential studies we identify as a result of the search strategy. We will resolve any disagreement through discussion or, if required, we will consult a third review author.

\section{Data extraction and management}

We will design a form to extract data. For eligible studies, two review authors will extract the data using the agreed form. We will resolve discrepancies through discussion or, if required, we will consult a third review author. We will enter data into Review Manager software (RevMan 2014) and check for accuracy.

When information regarding any of the above is unclear, we will attempt to contact authors of the original reports to provide further details.

\section{Assessment of risk of bias in included studies}

Two review authors will independently assess risk of bias for each study using the criteria outlined in the Cochrane Handbook for Systematic Reviews of Interventions ( Higgins 2011). We will resolve any disagreement by discussion or by involving a third assessor.

\section{(1) Random sequence generation (checking for possible selection bias)}

We will describe for each included study the method used to generate the allocation sequence in sufficient detail to allow an assessment of whether it should produce comparable groups.

We will assess the method as:

- low risk of bias (any truly random process, e.g. random number table; computer random number generator);

- high risk of bias (any non-random process, e.g. odd or even date of birth; hospital or clinic record number);

- unclear risk of bias.

\section{(2) Allocation concealment (checking for possible selection bias)}

We will describe for each included study the method used to conceal allocation to interventions prior to assignment and will assess whether intervention allocation could have been foreseen in advance of, or during recruitment, or changed after assignment.

We will assess the methods as:

- low risk of bias (e.g. telephone or central randomisation; consecutively numbered sealed opaque envelopes);

- high risk of bias (open random allocation; unsealed or non-opaque envelopes, alternation; date of birth);

- unclear risk of bias. 


\section{(3.1) Blinding of participants and personnel (checking for possible performance bias)}

We will describe for each included study the methods used, if any, to blind study participants and personnel from knowledge of which intervention a participant received. We will consider that studies are at low risk of bias if they were blinded, or if we judge that the lack of blinding would be unlikely to affect results. We will assess blinding separately for different outcomes or classes of outcomes.

We will assess the methods as:

- low, high or unclear risk of bias for participants;

- low, high or unclear risk of bias for personnel.

\section{(3.2) Blinding of outcome assessment (checking for possible detection bias)}

We will describe for each included study the methods used, if any, to blind outcome assessors from knowledge of which intervention a participant received. We will assess blinding separately for different outcomes or classes of outcomes.

We will assess methods used to blind outcome assessment as:

- low, high or unclear risk of bias.

\section{(4) Incomplete outcome data (checking for possible attrition bias due to the amount, nature and handling of incomplete outcome data)}

We will describe for each included study, and for each outcome or class of outcomes, the completeness of data including attrition and exclusions from the analysis. We will state whether attrition and exclusions were reported and the numbers included in the analysis at each stage (compared with the total randomised participants), reasons for attrition or exclusion where reported, and whether missing data were balanced across groups or were related to outcomes. Where sufficient information is reported, or can be supplied by the trial authors, we will re-include missing data in the analyses which we undertake.

We will assess methods as:

- low risk of bias (e.g. no missing outcome data; missing outcome data balanced across groups);

- high risk of bias (e.g. numbers or reasons for missing data imbalanced across groups; 'as treated' analysis done with substantial departure of intervention received from that assigned at randomisation);

- unclear risk of bias.

\section{(5) Selective reporting (checking for reporting bias)}

We will describe for each included study how we investigated the possibility of selective outcome reporting bias and what we found.

We will assess the methods as:

- low risk of bias (where it is clear that all of the study's pre-specified outcomes and all expected outcomes of interest to the review have been reported);

- high risk of bias (where not all the study's pre-specified outcomes have been reported; one or more reported primary outcomes were not pre-specified; outcomes of interest are reported incompletely and so cannot be used; study fails to include results of a key outcome that would have been expected to have been reported);

- unclear risk of bias.

\section{(6) Other bias (checking for bias due to problems not covered by (1) to (5) above)}

We will describe for each included study any important concerns we have about other possible sources of bias.

We will assess whether each study was free of other problems that could put it at risk of bias:

- low risk of other bias;

- high risk of other bias;

- unclear whether there is risk of other bias.

\section{(7) Overall risk of bias}

We will make explicit judgements about whether studies are at high risk of bias, according to the criteria given in the Cochrane Handbook (Higgins 2011). With reference to (1) to (6) above, we will assess the likely magnitude and direction of the bias and whether we consider it is likely to impact on the findings. We will explore the impact of the level of bias through undertaking sensitivity analyses - see Sensitivity analysis. 


\section{Assessing the quality of the evidence using the GRADE approach}

We will use the GRADE approach (as outlined in the GRADE handbook) in order to assess the quality of the body of evidence relating to the following outcomes for the main comparisons.

\section{For the infant/child}

- Death or any neurosensory disability (at latest time reported) (this combined outcome recognises the potential for competing risks of death or survival with neurological problems)

- Death (defined as all fetal, neonatal or later death) (at latest time reported)

- Neurosensory disability (any of cerebral palsy, blindness, deafness, developmental delay/intellectual impairment) (at latest time reported)

- Active resuscitation via an endotracheal tube at birth

- Admission to neonatal intensive care

- Neonatal encephalopathy

\section{For the mother}

- Any adverse effects severe enough to stop treatment.

We will use the GRADEpro Guideline Development Tool to import data from Review Manager 5.3 (RevMan 2014) in order to create 'Summary of findings' tables. A summary of the intervention effect and a measure of quality for each of the above outcomes will be produced using the GRADE approach. The GRADE approach uses five considerations (study limitations, consistency of effect, imprecision, indirectness and publication bias) to assess the quality of the body of evidence for each outcome. The evidence can be downgraded from 'high quality' by one level for serious (or by two levels for very serious) limitations, depending on assessments for risk of bias, indirectness of evidence, serious inconsistency, imprecision of effect estimates or potential publication bias.

\section{Measures of treatment effect}

\section{Dichotomous data}

For dichotomous data, we will present results as summary risk ratio with $95 \%$ confidence intervals.

\section{Continuous data}

For continuous data, we will use the mean difference if outcomes are measured in the same way between trials. We will use the standardised mean difference to combine trials that measure the same outcome, but use different methods.

\section{Unit of analysis issues}

\section{Cluster-randomised trials}

We consider cluster-randomised trials as inappropriate for this research question.

\section{Cross-over trials}

We consider cross-over trials as inappropriate for this research question.

\section{Multiple pregnancies}

As infants from multiple pregnancies are not independent, we plan to use cluster trial methods in the analyses, where the data allow, and where multiples make up a substantial proportion of the trial population, to account for non-independence of variables (Gates 2004).

\section{Multi-armed studies}

If multi-armed studies are included in the review, we plan to combine groups where appropriate in order to create a single pair-wise comparison (e.g. melatonin versus alternative neuroprotective treatments).

If an included trial has an intervention arm that is not relevant to the review question, we will comment on this in the table of 'Characteristics of included studies', and include in the review only the intervention and control groups that meet the eligibility criteria.

\section{Dealing with missing data}

For included studies, we will note levels of attrition. We will explore the impact of including studies with high levels of missing data in the overall assessment of treatment effect by using sensitivity analysis.

For all outcomes, we will carry out analyses, as far as possible, on an intention-to-treat basis, i.e. we will attempt to include all participants randomised to each group in the analyses, and all participants will be analysed in the group to which they were allocated, regardless of 
whether or not they received the allocated intervention. The denominator for each outcome in each trial will be the number randomised minus any participants whose outcomes are known to be missing.

\section{Assessment of heterogeneity}

We will assess statistical heterogeneity in each meta-analysis using the $\mathrm{T}^{2}, \mathrm{I}^{2}$ and $\mathrm{Chi}^{2}$ statistics. We will regard heterogeneity as substantial if an $\mathrm{I}^{2}$ is greater than $30 \%$ and either $\mathrm{T}^{2}$ is greater than zero, or there is a low $\mathrm{P}$ value (less than 0.10 ) in the Chi ${ }^{2}$ test for heterogeneity.

\section{Assessment of reporting biases}

If there are 10 or more studies in the meta-analysis we will investigate reporting biases (such as publication bias) using funnel plots. We will assess funnel plot asymmetry visually. If asymmetry is suggested by a visual assessment, we will perform exploratory analyses to investigate it.

\section{Data synthesis}

We will carry out statistical analysis using the Review Manager software (RevMan 2014). We will use fixed-effect meta-analysis for combining data where it is reasonable to assume that studies are estimating the same underlying treatment effect: i.e. where trials are examining the same intervention, and the trials' populations and methods are judged sufficiently similar. If there is clinical heterogeneity sufficient to expect that the underlying treatment effects differ between trials, or if substantial statistical heterogeneity is detected, we will use randomeffects meta-analysis to produce an overall summary, if an average treatment effect across trials is considered clinically meaningful. The random-effects summary will be treated as the average range of possible treatment effects and we will discuss the clinical implications of treatment effects differing between trials. If the average treatment effect is not clinically meaningful, we will not combine trials.

If we use random-effects analyses, the results will be presented as the average treatment effect with $95 \%$ confidence intervals, and the estimates of $\mathrm{T}^{2}$ and $\mathrm{I}^{2}$.

\section{Subgroup analysis and investigation of heterogeneity}

We will perform separate comparisons for those trials comparing melatonin with no treatment or a placebo, and those comparing melatonin with an alternative neuroprotective agent.

If we identify substantial heterogeneity, we will investigate it using subgroup analyses and sensitivity analyses. We will consider whether an overall summary is meaningful, and if it is, use random-effects analysis to produce it.

Maternal characteristics, and characteristics of the intervention, are likely to affect health outcomes. We will carry out subgroup analyses, if sufficient data are available, based on:

- gestational age at which the woman commenced melatonin treatment (e.g. $<26$ weeks versus 26 to $<28$ weeks versus 28 to $<30$ weeks versus 30 to $<32$ weeks versus 32 to $<34$ versus 34 to $<37$ weeks versus 37 weeks and over);

- reasons the mother was considered for melatonin treatment (e.g. preterm versus growth-restricted fetus versus prolonged prelabour rupture of membrane versus increased risk of chorioamnionitis versus pre-eclampsia/eclampsia versus increased risk of perinatal asphyxia versus other);

- total daily dose of melatonin administered (e.g. low ( $\leq 10 \mathrm{mg}$ daily) versus moderate ( $\leq 40 \mathrm{mg}$ daily) versus high ( $>70 \mathrm{mg}$ daily));

- mode of administration (e.g. intramuscular versus intravenous versus oral);

- number of babies in utero (e.g. singleton versus multiple).

We will use primary outcomes in subgroup analyses.

We will assess subgroup differences by interaction tests available within RevMan (RevMan 2014). We will report the results of subgroup analyses quoting the $\times 2$ statistic and $P$ value, and the interaction test $I^{2}$ value.

\section{Sensitivity analysis}

We will carry out sensitivity analysis to explore the effects of trial quality assessed by allocation concealment and other risk of bias components, by omitting studies rated as 'high risk of bias' for these components. We will restrict this to the primary outcomes.

\section{CONTRIBUTIONSOF AUTHORS}

Emily Bain drafted the first version of this review, with Dominic Wilkinson and Euan Wallace making comments and contributing to the final draft.

Emily Bain drafted the first version of the protocol for this review, with Dominic Wilkinson and Euan Wallace making comments and contributing to the final draft. 


\section{DECLARATIONS OF INTEREST}

Euan Wallace is a principal investigator on the MEL and the PAMPR trials (clinical trials of melatonin in women with an IUGR fetus or earlyonset preeclampsia) which may be considered for inclusion in this review in future updates. Therefore, assessment of eligibly of these trials, and if included, data collection and analysis (including 'Risk of bias' assessment) for these trials, will be carried out by the other two review authors (Dominic Wilkinson and Emily Bain) who are not directly involved in these trials. He is also co-investigator for an NHMRC application for a Program Grant in Perinatal Medicine which will include a future trial of melatonin in IUGR.

Dominic Wilkinson: has received an early career fellowship from the Australian National Health and Medical Research Council (NHMRC). He has also received a project grant from NHMRC to carry out a research project: Magnesium sulphate at 30 to 34 weeks' gestational age: neuroprotection trial.

Emily Bain: None known

\section{SOURCES OF SUPPORT}

\section{Internal sources}

- ARCH: Australian Research Centre for Health of Women and Babies, Robinson Research Institute, Discipline of Obstetrics and Gynaecology, The University of Adelaide, Australia.

\section{External sources}

- National Health and Medical Research Council, Australia.

\section{DIFFERENCES BETWEEN PROTOCOL AND REVIEW}

None known.

\section{N DEX TERMS}

\section{Medical Subject Headings (MeSH)}

${ }^{\star}$ Neuroprotection; Fetus [ ${ }^{\star}$ drug effects]; Melatonin [ ${ }^{*}$ administration \& dosage]; Neuroprotective Agents [*administration \& dosage]

\section{MeSH check words}

Female; Humans; Pregnancy 